\title{
Primordial Rhythmic Bursting in Embryonic Cochlear Ganglion Cells
}

\author{
Timothy A. Jones, Sherri M. Jones, and Kristina C. Paggett \\ Department of Surgery, Division of Otolaryngology, University of Missouri School of Medicine, Columbia, Missouri 65212
}

This study examined the nature of spontaneous discharge patterns in cochlear ganglion cells in embryonic day 13 (E13) to early E17 chicken embryos (stages 39-43). Neural recordings were made with glass micropipettes. No sound-driven activity was seen for the youngest embryos (maximum intensity $107 \mathrm{~dB}$ sound pressure level). Ganglion cells were labeled with biotinylated dextran amine in four embryos. In two animals, primary afferents projected to hair cells in the middle region along the length of the basilar papilla in which, in one cell, the terminals occupied a neural transverse position and, in the other, a more abneural location. Statoacoustic ganglion cells showing no spontaneous activity were seen for the first time in the chicken. The proportion of "silent" cells was largest at the youngest stages (stage 39, 67\%). In active cells, mean spontaneous discharge rates [9.4 \pm 10.4 spikes $(\mathrm{Sp}) / \mathrm{sec} ; n=44]$ were lower than rates for older embryos (19 $\pm 17 \mathrm{Sp} / \mathrm{sec}$ ) (Jones and
Jones, 2000). Embryos at stages 39-41 evidenced even lower rates $(4.2 \pm 5.0 \mathrm{Sp} / \mathrm{sec})$. The most salient feature of spontaneous activity for stages 39-43 was a bursting discharge pattern in $>75 \%$ of active neurons (33 of 44 ). Moreover, in $55 \%$ of these cells, there was a clear, slow, rhythmic bursting pattern. The proportion of cells showing rhythmic bursting was greatest at the youngest stages (39-42) and decreased to $<30 \%$ at stage 43. Rate of bursting ranged from 1 to 54 bursts per minute. The presence of rhythmic bursting in cochlear ganglion cells at E13-E17 provides an explanation for the existence of such patterns in central auditory relays. The bursting patterns may serve as a patterning signal for central synaptic refinements in the auditory system during development.

Key words: functional ontogeny; bird; spontaneous activity; embryonic development; primary afferents; chicken; audition; hearing
Spontaneous rhythmic bursting of retinal ganglion cells may provide a critical signal for refinements in developing central binocular processing circuits. Indeed, modifications of retinal spontaneous afferent discharge patterns can result in altered synaptic configurations in thalamic and cortical relays (Galli and Maffei, 1988; Constantine-Paton et al., 1990; Shatz, 1990, 1996; Meister et al., 1991; Wong et al., 1993, 1995; Mooney et al., 1996; Masland, 1997).

Could development of binaural processing circuits require a patterned activity similar to rhythmic retinal activity? Unfortunately, little is known about spontaneous activity derived from the embryonic or neonatal cochlea. There are a few descriptions of spontaneous discharge patterns in developing central and peripheral auditory circuits. Low spontaneous discharge rates were described in the neonatal pigeon (Richter et al., 1996) and cat (Romand, 1984), but bursting patterns were not reported. Jones and Jones (2000) recorded single unit activity for primary afferents of the statoacoustic ganglion in chicken embryos at $19 \mathrm{~d}$ of incubation [embryonic day 19 (E19)]. These investigators demonstrated lower overall discharge rates for auditory and nonauditory primary afferents in the embryo as well as longer modes and dead time. Irregular bursting patterns were observed in $\sim 30 \%$ of embryonic neurons. However, no rhythmic bursting patterns were found except those occasionally driven by the

Received July 28, 2000; revised July 17, 2001; accepted July 27, 2001.

This research was supported by National Institutes of Health and National Institute on Deafness and Other Communication Disorders Grant R01 DC02753.

Correspondence should be addressed to Dr. Timothy A. Jones, Department of Surgery/Otolaryngology, University of Missouri School of Medicine, Room 202 Allton Building, DC375.00, 301 Business Loop 70W, Columbia, MO 65212. E-mail: JonesT@health.missouri.edu.

Copyright (C) 2001 Society for Neuroscience $\quad 0270-6474 / 01 / 218129-07 \$ 15.00 / 0$ heartbeat. Lippe (1994, 1995) characterized patterns of central multiunit activity in the region of brainstem relay nuclei in chicken embryos at ages between 14 and 19 d of incubation (E14 and E19). In addition to low overall discharge rates, Lippe described regular bursting patterns reminiscent of early bursting patterns that were reported for other sensory modalities, especially the visual system. Furthermore, he described the disappearance of central bursting patterns when cochlear input was eliminated, suggesting that central bursting depended in some way on primary afferents. Gummer and Mark (1994) also described “patterned neural activity" in brainstem auditory centers of the wallaby neonate. Although suggestive, observations to date provide no definitive evidence for a dominant regular bursting pattern in developing primary afferents of the cochlea. The present study examined activity patterns of cochlear ganglion cells at ages younger than E19.

\section{MATERIALS AND METHODS}

Methods for in vivo recordings of the statoacoustic ganglion in chicken embryos have been published elsewhere (Jones and Jones, 1995a,b, 2000). Briefly, embryos (Gallus domesticus) were incubated in commercial incubators at $37.5^{\circ} \mathrm{C}$ (65\% humidity) and were retrieved from incubators after 15-18 d. Animals were anesthetized with EquiThesin/saline $(1: 5 ; 0.1 \mathrm{ml})$. EquiThesin was administered subcutaneously at the neck, along with $1 \mathrm{mg}$ of gallamine triethiodide. Needle electrodes were used to monitor the electrocardiogram. The head was secured to a custom platform, and the scala tympani was exposed using a posterolateral approach. Brain temperature was monitored and maintained on average at $37.4 \pm 1.4^{\circ} \mathrm{C}$. Egg temperature was also monitored and ranged between 38 and $40^{\circ} \mathrm{C}$.

Beak and toe lengths were measured and used to determine the developmental stage of each animal. Ages are reported as stages or corresponding days of incubation as outlined by Hamburger and Hamilton (1951). Stages of development reported herein encompassed 39-43 


\begin{tabular}{ll}
\hline $\begin{array}{l}\text { Table 1. Staging and incubation day equivalence, according to } \\
\text { Hamburger and Hamilton (1951) }\end{array}$ \\
$\begin{array}{ll}\text { Equivalent incubation day } \\
\left(37.5^{\circ} \mathrm{C}\right)\end{array}$ \\
\hline 38 to 39 & $\mathrm{E} 12$ \\
$38+$ to $40-$ & $\mathrm{E} 13$ \\
$40-$ to 40 & $\mathrm{E} 14$ \\
$40-$ to 41 & $\mathrm{E} 15$ \\
$41+$ to 42 & $\mathrm{E} 16$ \\
42 to 43 & $\mathrm{E} 17$ \\
42 to 44 & $\mathrm{E} 18$
\end{tabular}

+ , Late; -, early.

(E13-E17). The University of Missouri Institutional Animal Care and Use Committee approved the care and use of the animals described in this report. Table 1 provides a summary of developmental stages and equivalent incubation days.

Beveled glass micropipettes, filled with $10 \%$ biotinylated dextran amine (BDA; 3000 molecular weight; Molecular Probes, Eugene, OR) in $0.5 \mathrm{M} \mathrm{KCl}$ and $0.05 \mathrm{M}$ Tris, were lowered into scala tympani in $0.5 \mu \mathrm{m}$ steps with the tip directed toward the ganglion. Silver chloride wire electrodes were used for the reference (neck) and ground (extraembryonic fluid). Electrode impedance ranged from 5 to $15 \mathrm{M} \Omega$ and was measured in situ using an electrometer (WPI 767-B; World Precision Instruments, Sarasota, FL).

Electrophysiological activity was amplified (gains ranged from 50 to $500 \times$ ), filtered (bandpass $100-3000 \mathrm{~Hz}$, or $100-10,000 \mathrm{~Hz},-6 \mathrm{~dB}$ points), and recorded on videotape (Vetter 420G). Signals were also amplified and led to a speaker so that the activity of neurons was audible. Detailed analysis of spontaneous activity was accomplished off-line. Activity was digitized (40 or $50 \mu \mathrm{sec} /$ point) and saved on diskette. Then, the recording was systematically analyzed to determine the onset time of each spike occurrence. A voltage threshold criterion was used to detect spikes initially, and each spike detected was displayed at high resolution and visually confirmed on screen (Fig. 1). In Figure 1, records from two neurons illustrate the range of signal-to-noise ratios found typically in the present study.

Fluid was wicked out of the middle ear, and sound stimuli were delivered using an ER-2 earphone that was sealed at the external auditory meatus. Clicks ( $0.1 \mathrm{msec} ; 1.0 \mathrm{~V}$ square wave pulses), pure tones (1.0 $\mathrm{V}$ peak to peak; $100-4000 \mathrm{~Hz})$, and pure-tone or noise bursts $(50 \mathrm{msec}$ duration; 5 msec rise-fall times; $50 \%$ duty cycle) were used as stimuli to determine whether individual cells responded to sound. All stimuli were routed through an attenuator (Med Associates, St. Albans, Vermont), amplifier (Med Associates), and equalizer (Pioneer, Long Beach, CA) before output at the earphone. Maximum stimulus intensity at the output of the earphone was $107 \mathrm{~dB}$ sound pressure level (SPL) (or dB peak equivalent SPL for clicks across the frequencies tested).

Once single primary afferents were isolated, up to $4 \mathrm{~min}$ of continuous spontaneous activity was recorded on analog tape and analyzed off-line. If the neuron could be sound activated, an estimate of best frequency was obtained audiovisually (Jones and Jones, 1995a,b) using manually swept pure tones $(100-4000 \mathrm{~Hz})$ that were produced by a sine wave generator (Wavetek, Everett, WA). The tone frequencies producing the largest audible response were noted and recorded as an estimate of best frequency. It was possible in some cases to estimate the sound threshold at best frequency. To do this, stimulus frequency was swept across the best frequency (from above and below) while the sound level of the stimulus was adjusted to determine the lowest sound level producing an audible increase in firing.

We use the expression "rhythmic bursting" here to describe recurring periodic and generally sudden increases in neural discharge rate. When present, bursts of activity are heralded by a flurry of spikes (at least two in number, but usually many more) separated by relatively long silent periods that reoccur at somewhat regular intervals. The striking character of ideal regular bursting is easily recognized. However, in the case of less than ideal rhythmic bursting patterns or less prevalent long silent periods, (as is the case in late embryonic periods) it becomes harder to characterize the regularity and hence the rate of bursting. We addressed this issue in the present study by evaluating spike trains using autocorrelation and the fast Fourier transform (FFT). As described in earlier
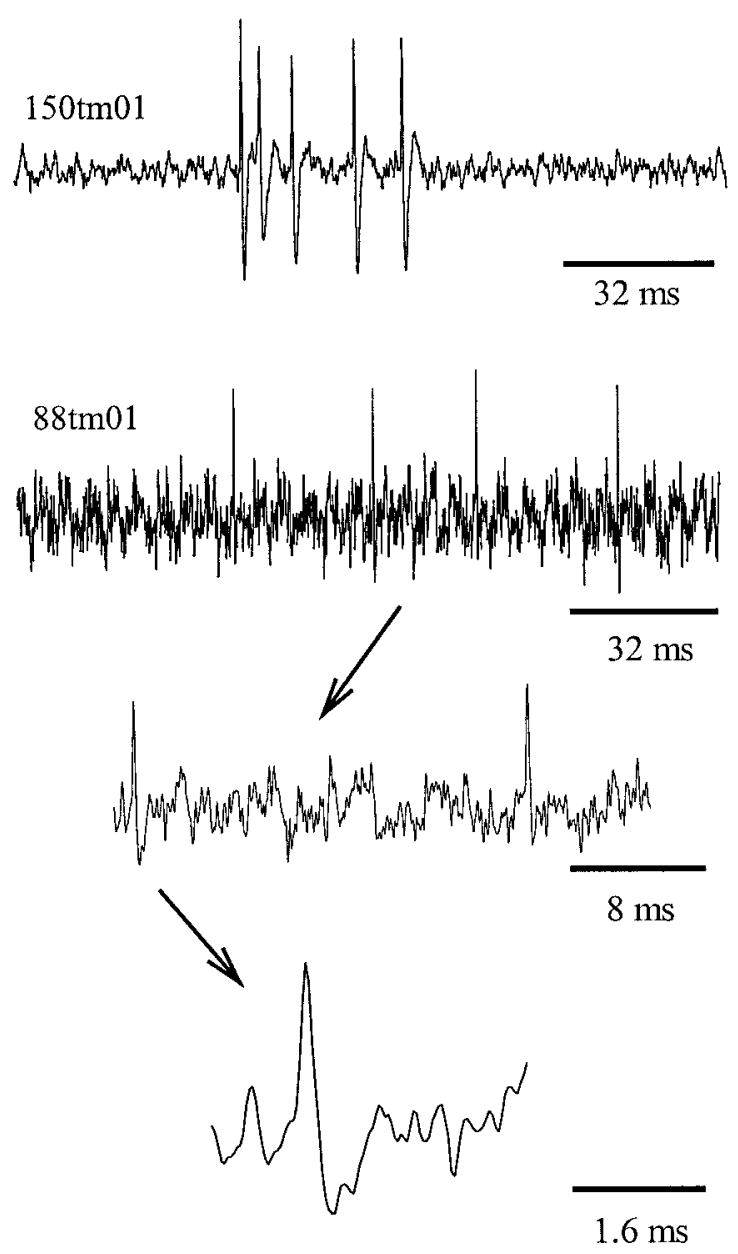

Figure 1. The primary digitized records (40 $\mu$ sec per point) of two neural spike trains are shown here. The records illustrate the range of signal-to-noise ratios encountered in this study. The process of expanding traces for high resolution computerized analysis is shown for neuron $88 \mathrm{tm} 01$. Spike detection was accomplished using an initial voltage threshold criterion. Records were divided into contiguous data blocks in which for each block, the voltage threshold could be adjusted to accommodate fluctuations in baseline. The entire record was examined on screen at high resolution for falsely detected spikes and for failed detection of spikes. Each action potential was individually confirmed.

work (Jones and Jones, 2000), probability density functions (autocorrelation functions, ACFs) were produced off-line using the strategies outlined by Moller, (1970). Bin size was 9 or $30 \mathrm{msec}$, and 1024 bins were used to form the ACF array. Autocorrelation functions were calculated and then subjected to Fourier analysis to identify the fundamental frequency $\left(\mathrm{f}_{0}\right)$ of rhythmic components. The spectral fundamental frequency provided a quantitative estimate of the rhythmic discharge rate (i.e., burst rate). The FFT spectrum also provided a means to judge the strength of rhythmicity, or whether rhythmic bursting was considered present or not.

Spontaneous discharge patterns not showing rhythmic bursting based on the ACF were also tested for levels of irregular bursting. Irregular bursting patterns are characterized by bouts of neural activity that occur at irregular intervals separated by relatively long periods of silence. To objectively quantify the level of irregular bursting activity ("burstiness"), we used a numerical "burst factor" (BF) as described in a previous report (Jones and Jones, 2000). The BF has two principal computational components, $A$ and $B$. The number $A$ is proportional to the relative amount of time a cell spends in long silent periods. The more time spent in long silent periods, the greater the burst factor. Component $A$ was calculated by summing the duration of the longest silent periods (i.e., the longest spike intervals) and then expressing the sum as a percentage of the entire recording sample. During analysis, spike intervals were sorted to identify 
the longest silent periods. It was necessary to determine the number of longest silent periods to use in generating the sum. This number is designated as $C$, and its value is calculated using the equation $C=5 \%$ of the total number of intervals or 4 , whichever was largest. Component $B$ provides an adjustment for the relative amount of activity present during bursts of activity, such that the greater the contrast between silent and active periods, the larger the BF. $B$ was defined as the ratio of the mean spike interval of the longest silent periods (as defined above) divided by the mean spike interval over the entire sample. $B$ therefore will tend to be largest when long silent periods are accompanied by robust active periods having high discharge rates. $\mathrm{BF}$ is the product of the two components, $A$ and $B\left(\mathrm{BF}=A^{*} B\right)$, where $A=[($ total time of the longest $C$ intervals $) /($ total sample time $)]$ and $B=[($ mean interval length of the longest $C$ intervals)/(total sample time/total number of intervals)]. The manner in which $C$ was determined here differed slightly from our earlier study (using a percentage vs a constant number of 4). The method used here is more general and serves to accommodate the very long recordings made here. The BF provides a metric to identify and rank irregular bursting patterns. In previous work, values for BF ranged between 0 and 10 for embryos. In contrast, BF values in post-hatch animals ranged between 0 and 1 . Auditory neurons in post-hatch animals demonstrate no irregular bursting patterns. Instead, spontaneous activity appears as a relatively continuous stochastic process. $\mathrm{BF}$ values $>1$ reflect the presence of bursting in which the greater the $\mathrm{BF}$, the more pronounced is the bursting. We arbitrarily set a BF value of 1.1 as cutoff level for bursting. Neural discharge patterns generating a BF of 1.1 or greater are categorized as bursting patterns.

After physiological recordings, BDA was ejected from the pipette using pressure $(\sim 300 \mathrm{kPa})$. One to two hours after the injection, the cochlea was fixed and processed. Several fixation and processing protocols were used to increase the likelihood of successful label. The following protocol has thus far been the most successful. The tympanic membrane was opened, the columella was removed, and the cochlea was perfused slowly with $3 \mathrm{ml}$ of $4 \%$ paraformaldehyde $/ 1 \%$ glutaraldehyde in $0.1 \mathrm{~m}$ phosphate buffer. Immediately after cochlear perfusion, the embryo was decapitated, the head was hemisected, and an additional 3-5 ml of fixative was perfused into the opened labyrinth. The medial wall of the cochlea was also opened, and the head was stored in fixative overnight. Our experience suggests that initial perfusion of the cochlea must occur in an intact viable animal. We have not seen successful label in any case in which cochlear perfusion was initiated in a physiologically compromised animal (slow or irregular electrocardiogram or immediately after decapitation).

Dissected cochleas were processed as whole mounts. Processing began with four rinses in PBS (10 min each). The final PBS rinse contained 1\% Triton X-100. Rinses were followed with pretreatment in $0.5 \% \mathrm{H}_{2} \mathrm{O}_{2}$ in PBS (20 min to block endogenous peroxidase) and three additional rinses in PBS (10 min each). Then, cochleas were soaked overnight in avidinHRP (Vector Laboratories, Burlingame, CA):PBS containing 1\% Triton X-100 (1:250). The next day, cochleas were rinsed again with PBS (three times, $10 \mathrm{~min}$ each) and soaked in $0.03 \% \mathrm{DAB}$ for $20-30 \mathrm{~min}$. At this point, $0.03 \% \mathrm{H}_{2} \mathrm{O}_{2}$ was added, one drop every 1-2 min until desired background stain was achieved (usually an additional 5-8 min). Processed cochleas were embedded in plastic (JB-4) and viewed with light microscopy.

\section{RESULTS}

Data used for analysis were obtained from embryos in good physiological condition (i.e., stable brain temperatures and regular heart rate $>250$ beats per minute). Studies were terminated, or data were excluded for embryos that did not meet these criteria because they were judged to be physiologically compromised. Sixty-one statoacoustic ganglion cells were isolated for study in 51 embryos. All but 17 of the neurons were spontaneously active. The 17 "silent" neurons produced trains of action potentials with current injections ranging from 0.1 to $25 \mathrm{nA}$.

Discharge rates [spikes (Sp) per second] for spontaneously active individual ganglion cells are plotted as a function of developmental stage in Figure 2. Rates were based on recordings with an average duration of $\sim 87 \mathrm{sec}$, with the lowest rate based on 8 spikes in $90 \mathrm{sec}$ and the highest rate based on 406 spikes in 10.23 sec. The total number of action potentials per record ranged from 8 to 2184 spikes. The longest continuous record of spontaneous

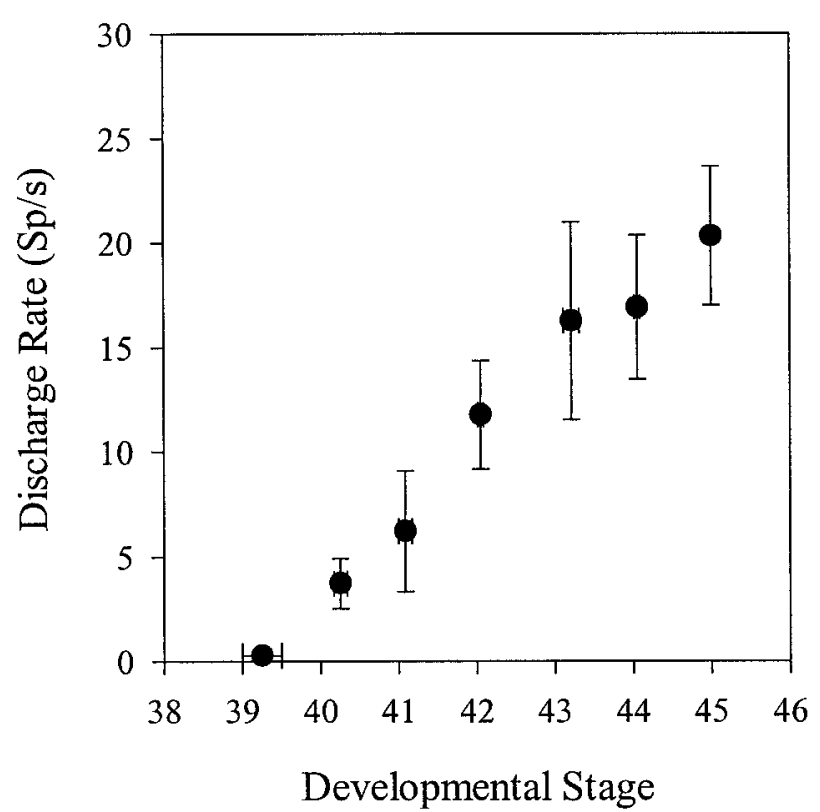

Figure 2. Discharge rates (spikes per second) for individual ganglion cells are plotted as a function of developmental stage. Data for stages 44 and 45 are from earlier work (Jones and Jones, 2000). Spike discharge rates increase steadily during development (regression slope $=4.13 \mathrm{Sp} / \mathrm{sec}$ per stage; $p<0.001 ; r^{2}=0.2$ ). Discharge rates for stages 39-43 ranged from 0.09 to $40 \mathrm{Sp} / \mathrm{sec}$, and the overall mean discharge rate was $9 \pm 10$ $\mathrm{Sp} / \mathrm{sec}(n=44)$.

activity was $4 \mathrm{~min}$. To establish a broader context, we have added data from earlier work for stages 44 and 45 (Jones and Jones, 2000). The range of spike discharge rates steadily increased during development. Mean spike rate increased from $0.27 \pm 0.26$ $\mathrm{Sp} / \mathrm{sec}(n=2)$ at stage 39 to $16.3 \pm 12.5 \mathrm{Sp} / \mathrm{sec}(n=7)$ at stage 43 (Fig. 2, linear regression slope, $4.13 \mathrm{Sp} / \mathrm{sec}$ per stage; $p<0.001$; $\left.r^{2}=0.2 ; n=44\right)$. Mean values at stages 41 and 42 were $6.2 \pm 7.1$ $\mathrm{Sp} / \mathrm{sec}(n=6)$ and $11.8 \pm 11.3 \mathrm{Sp} / \mathrm{sec}(n=19)$, respectively. Average spike rates for stage 43 approached rates published for stages 44-45 (19 $\pm 17 \mathrm{Sp} / \mathrm{sec}$, from Jones and Jones, 2000).

Five examples of discharge patterns from stages 39-43 are represented in Figure 3. These records reflect the slow rhythmic discharge patterns or bursting found in animals younger than E18. The frequency of bursting was generally quite low $(<1 \mathrm{~Hz}$, ranging from 0.0167 to $0.9 \mathrm{~Hz}$; mean $\pm \mathrm{SD}=0.27 \pm 0.19 ; n=$ 18). Figure 4 illustrates the results of autocorrelation and Fourier analysis on some of these neurons. Neuron $80 \mathrm{tm} 01$ demonstrates modest spike discharge rates overall $(3.9 \mathrm{Sp} / \mathrm{sec})$ and is featured at the top of Figure 4. The ACF array for $80 \mathrm{tm} 01$ reveals a strong periodicity in firing, whereas the FFT demonstrates a corresponding $\mathrm{f}_{0}$ of $0.228 \mathrm{~Hz}$. The burst rate in bursts per minute is calculated as $B R=f_{0} \times 60 \mathrm{sec} / \mathrm{min}$, or $\sim 13.4$ bursts per minute in this case. A second rhythmically bursting neuron from Figure 3 is shown in Figure 4 (91tm01). Despite relatively low overall spike rates $(0.45 \mathrm{Sp} / \mathrm{sec})$, the ACF reveals a clear periodicity and a significant FFT fundamental at $\mathrm{f}_{0}=0.163 \mathrm{~Hz}$ or $\sim 9.8$ bursts per minute. The neuron at the bottom of Figure 4 (76tm01) is an example in which rhythmic bursting is absent. No periodicity is apparent in the ACF, and the FFT has no significant peaks. This neuron, although not a rhythmically bursting cell, was classified as irregularly bursting, having a $\mathrm{BF}=1.99$. All neurons of Figure 3 had robust rhythmic patterns. There were examples of neurons in the present study that displayed evidence of very weak periodi- 

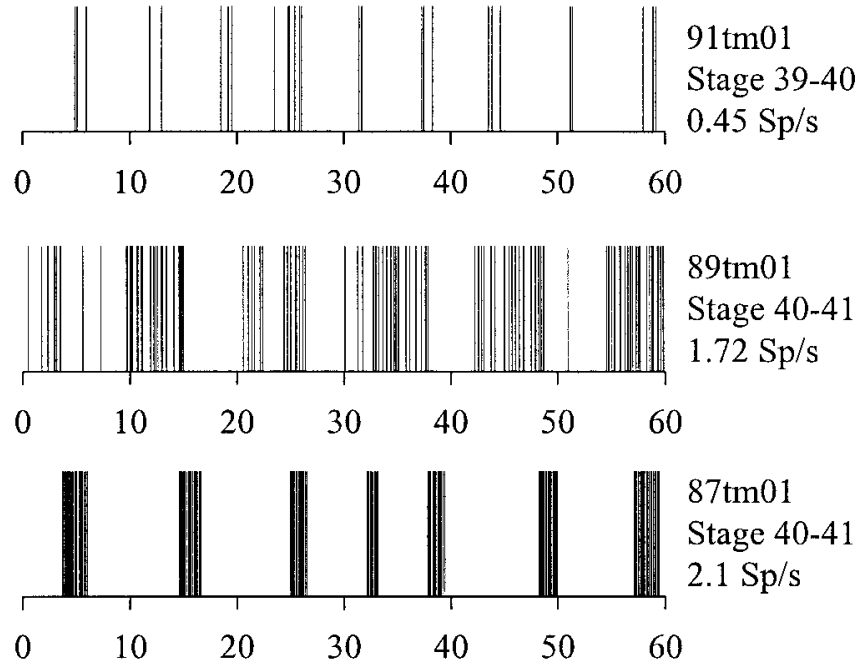

$\begin{array}{lllllll}0 & 10 & 20 & 30 & 40 & 50 & 60\end{array}$
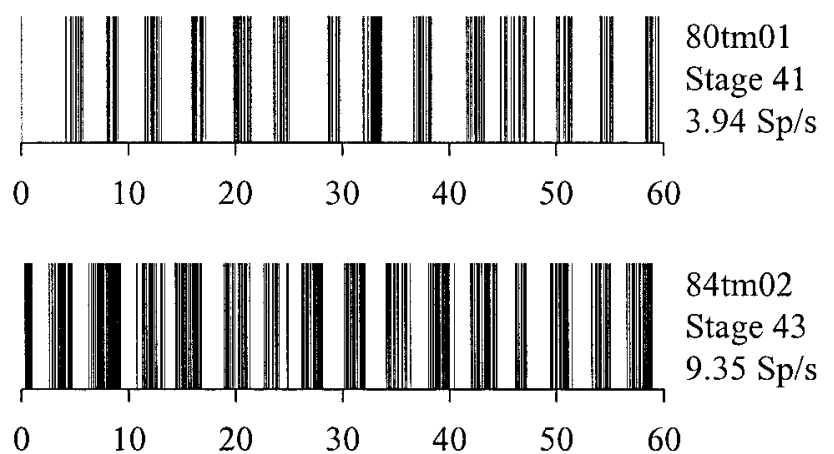

Time (Seconds)

Figure 3. Bursting spontaneous discharge patterns. Five examples of discharge patterns are represented at progressively later stages of development from stage 39 to 43 (top to bottom, respectively). Eighteen of the 44 spontaneously active ganglion cells produced rhythmic bursting patterns. Each vertical line represents a single action potential. Total time represented is $60 \mathrm{sec}$. Over these developmental stages, the rate of bursting ranged from 1 to 54 bursts per minute. Burst rates for each neuron were based on the FFT spectrum of the ACF (Fig. 4). Rates in bursts per minute for neurons shown were 9.78 for $91 \mathrm{tm} 01,5.88$ for $89 \mathrm{tm} 01,5.88$ for $87 \mathrm{tm} 01,13.68$ for $80 \mathrm{tm} 01$, and 15.6 for $84 \mathrm{tm} 02$. Five cells that demonstrated regular bursting patterns (all at stages 42-43) also responded to sound.

cities, and it was necessary to adopt an objective conservative criterion distinguishing rhythmic from nonrhythmic patterns. Activity patterns producing spectral peaks with magnitudes less than twice baseline levels were classified as nonrhythmic (irregular bursting). Those producing spectra with a clear fundamental frequency peak that was two or more times that of the background levels were judged to be regularly bursting neurons.

The average rhythmic burst rate (Fig. 5) increased during stages 39-43. Burst rate increased from 9.8 bursts per minute at stage 39 to 35 bursts per minute at stage 43 (linear regression slope, 5.68 bursts per minute per stage; $p<0.01 ; r^{2}=0.3 ; n=18$ ). In many cases, the bursting was quite regular and contrasted markedly with irregular bursting patterns described in detail for a substantial number of primary afferents in animals older than E18 (Jones and Jones, 2000). In all cases, bursting was unrelated to heart beat. Eighteen of the 44 spontaneously active ganglion cells produced rhythmic bursting patterns (e.g., Fig. 3). Five of the
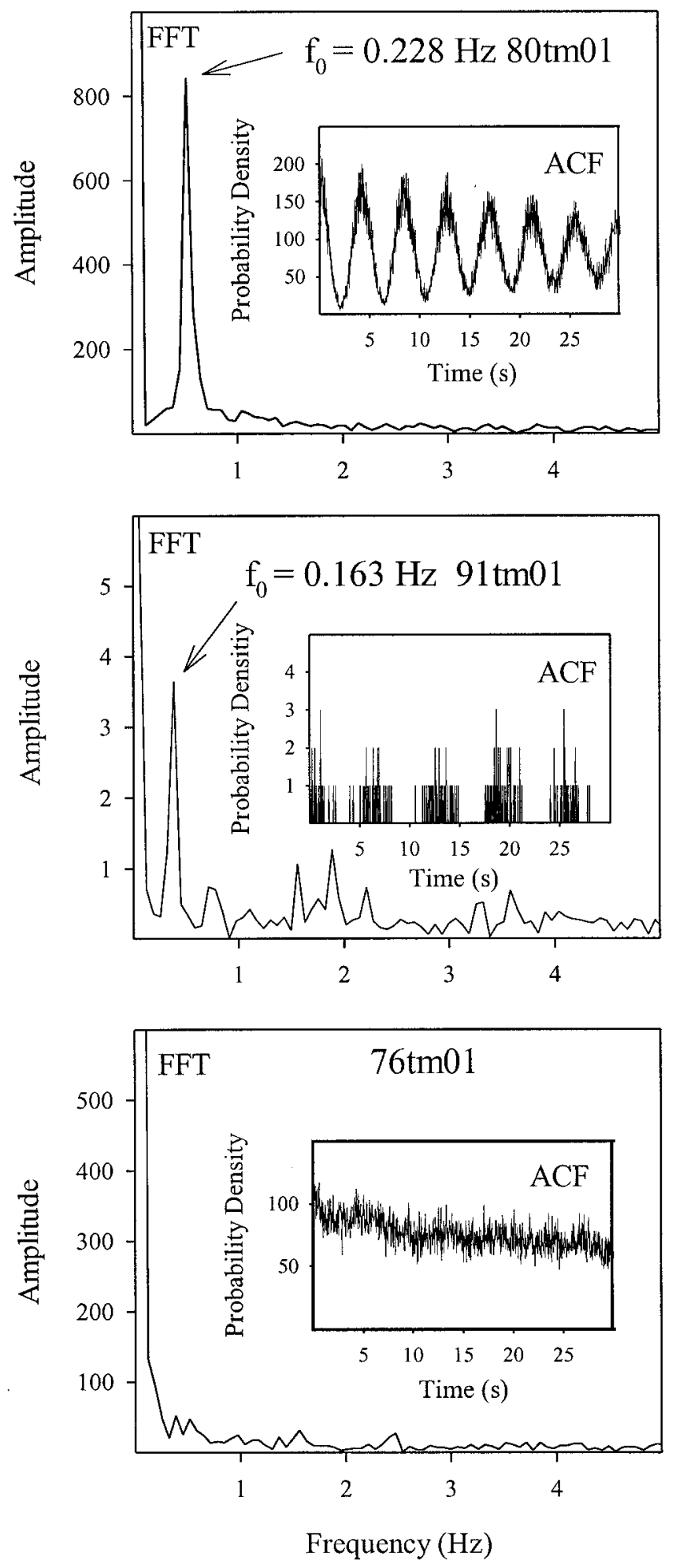

Figure 4. Autocorrelation functions ( $A C F$; also referred to as probability density functions) and their fast Fourier transforms (FFT) are shown for three representative neurons. The ACF of spike discharge activity was examined for evidence of periodicities. Neuron $80 \mathrm{tm} 01$ (top) shows a robust periodicity, and the FFT spectrum demonstrates a fundamental frequency $\left(f_{o}\right)$ of $0.228 \mathrm{~Hz}$. A portion of the spike train for neuron is shown in Figure 3 . Similarly, the spike activity of neuron $91 \mathrm{tm} 01$ of Figure 3 was subjected to ACF and FFT analysis, revealing a clear periodicity at $0.163 \mathrm{~Hz}$, despite the low spike rate in this case. Neuron 76tm01 exhibited no rhythmic bursting, and as can be seen in the ACF, there were no periodicities present. FFT amplitudes are represented in arbitrary units. 

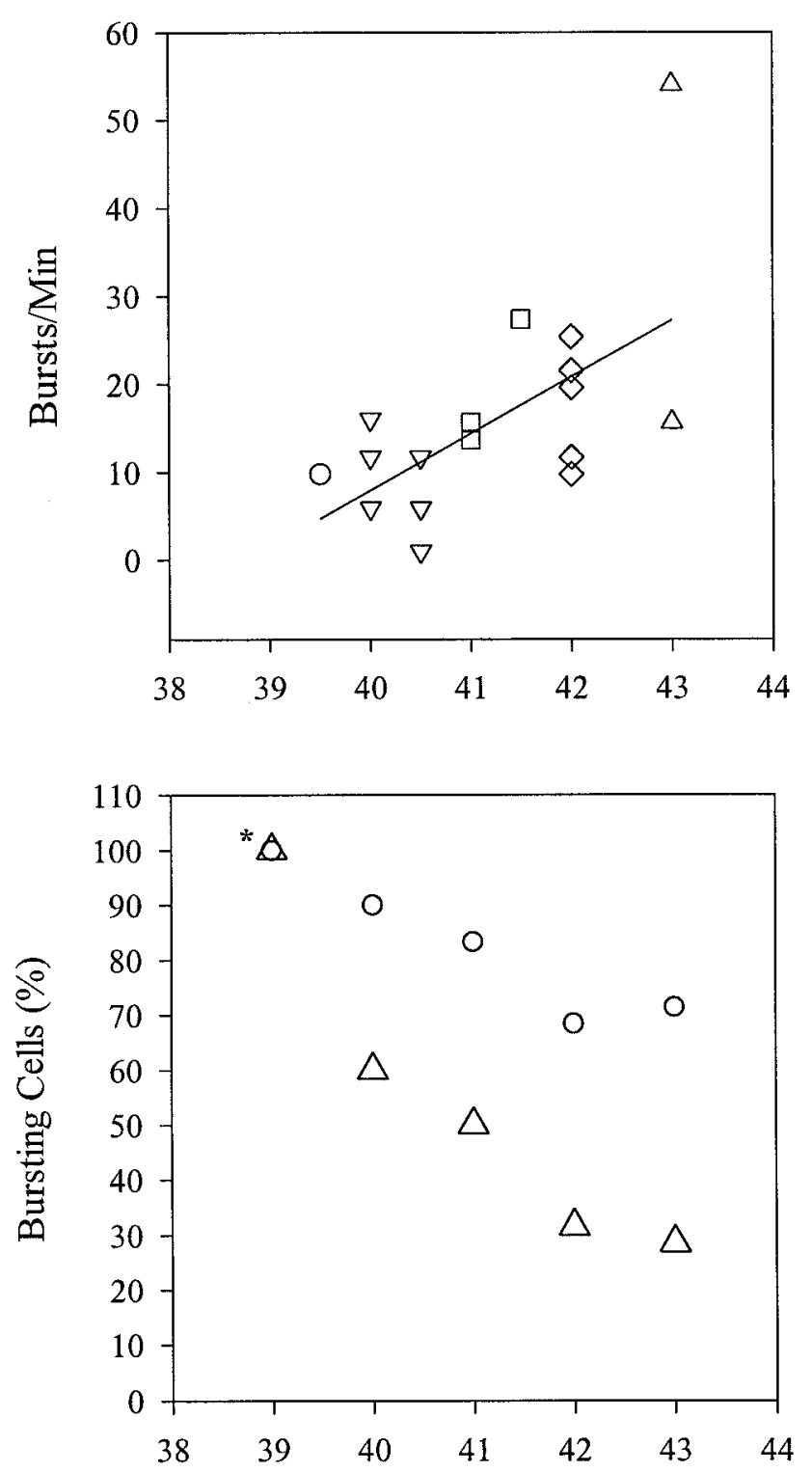

Developmental Stage

Figure 5. Rhythmic discharge rates (bursts per minute) are plotted as a function of developmental stage (top). Rate of rhythmic bursting increased on average with increasing developmental stage. The range of burst rates was 1-54 bursts per minute. The regression slope for data shown was 5.68 bursts per minute per stage $\left(p<0.01 ; r^{2}=0.3\right)$. At stages $40-43$, both rhythmic and irregular bursting patterns were present. Rates indicated here are for rhythmic bursting only. Bursting patterns are irregular when present at stages beyond 43 . The relative number of neurons exhibiting rhythmic bursting decreased with increasing stage (bottom). Circles represent both rhythmic and nonrhythmic bursting patterns, whereas triangles represent proportions of rhythmically bursting neurons only. The fraction of rhythmically bursting cells decreases at later stages. * indicates that in the case of stage 39 , most cells were silent. The two cells that were active had very low spike rates, and only one had a sufficient number of spikes to evaluate for bursting patterns. This cell exhibited rhythmic bursting. The other cell generated only eight spikes in $90 \mathrm{sec}$, thus precluding a determination of bursting status.

rhythmic bursters (all at stages 42-43) responded to sound and had best frequencies between 500 and $1500 \mathrm{~Hz}$. In three of these neurons, threshold estimates were observed at 36, 66, and $71 \mathrm{~dB}$ SPL. No significant correlation was found between the manually

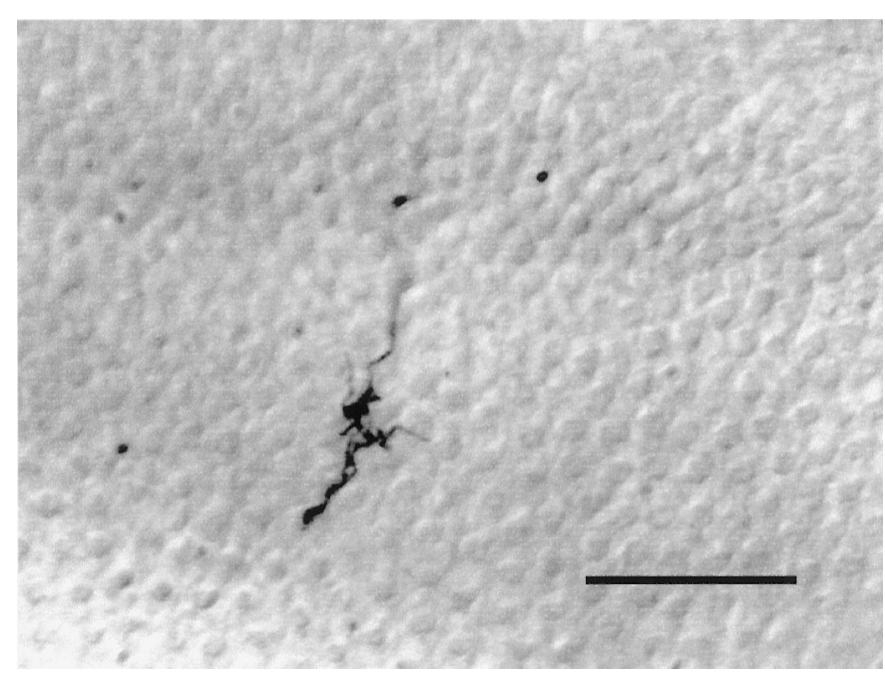

Figure 6. Labeled neuron for animal 87tm01. Spontaneous discharge pattern for this animal is shown in Figure 2. Top, The neural edge; bottom, the abneural edge; right, the base; and left, the apex. Scale bar, $50 \mu \mathrm{m}$.

determined best frequency and rate of bursting for these five neurons.

The relative percentage of neurons exhibiting spontaneous bursting changed as a function of developmental stage. At ages younger than stage 42,10 of the 18 active cells demonstrated rhythmic bursting. The relative number of rhythmically bursting cells decreased with increasing stage as illustrated in Figure 5 (bottom). There was a mixture of neurons displaying regular and irregular bursting patterns at stages 40-43. The proportion of silent cells also decreased progressively from $67 \%$ at stage 39 to zero at stages 44 and 45 . Figure 5 illustrates the relative number of irregularly and regularly bursting neurons at each stage. At stages $44-45$, bursting is evident in $\sim 33 \%$ of cells, and the bursting patterns are irregular (Jones and Jones, 2000). For the present data, interspike interval coefficient of variation $(\mathrm{CV}=$ $\mathrm{SD} /$ mean) was calculated for bursting and nonbursting neurons. The mean $\mathrm{CV}$ of bursting neurons was significantly higher (2.20 $\pm 1.12 ; n=33$; range, 1.17-7.22) than nonbursting cells (0.82 $\pm 0.34 ; n=10$; range, $0.15-1.21)$. Two of the nonbursting cells had time interval histograms with a Gaussian shape and CVs much $<0.5$. These latter discharge characteristics are normally seen with neurons innervating the lagena.

Cellular labeling was observed in four embryos. Figure 6 shows the primary afferent terminals for neuron $87 \mathrm{tm} 01$ (stage 40-41) of Figure 3. This neuron was located $50 \%$ of the distance from apex to base, it terminated toward the abneural edge of the papilla ( $60 \%$ of distance from the neural to abneural edge), it did not respond to sound at levels up to $103 \mathrm{~dB}$ SPL, and it generated the rhythmic discharge pattern shown in Figure 3. A second bursting neuron recorded from another embryo at stage 40-41 innervated a similar central region of the papilla but had terminals near the neural edge transversely $(10 \%$ of distance from neural to abneural edge). The label in the other two cases filled ganglion cell bodies but could not be resolved at the final terminals on hair cells.

\section{DISCUSSION}

The majority of embryonic ganglion cells at stages 39-43 (E13E17) display regular, rhythmic spontaneous bursting patterns. These data provide the first definitive evidence of primordial 
regular bursting patterns for primary afferents of the cochlear ganglion. The rate of bursting increases with age and becomes less regular as development proceeds. At stages beyond 43, only $33 \%$ of statoacoustic ganglion cells burst, and the bursting pattern is irregular (Jones and Jones, 2000). The presence of rhythmic bursting in cochlear ganglion cells at stages $40-43$ provides an explanation for the existence of such patterns in central auditory relays of embryos (Lippe, 1994, 1995). The range of rhythmic bursting rates found in the present study was somewhat wider than that reported by Lippe for comparable ages. Here, bursting rates increased significantly with age in ganglion cells, a finding that could explain a similar increase observed by Lippe in the brainstem. In contrast to Lippe, we found no relationship between best frequency and rate of bursting. The fact that we used a manual audiovisual procedure to estimate best frequency does not likely account for this difference because Lippe used a similar approach. On the other hand, we determined a best frequency in a relatively small number of cells, and it may be the case that larger numbers of neurons are required to resolve the putative relationship. This issue deserves further study.

Five of the rhythmically bursting cells of the present study responded to sound, thus identifying them as auditory fibers innervating the papilla. Two other rhythmically bursting cells did not respond to sound, but BDA labeling identified their cell bodies and showed them to innervate the papilla. These data clearly demonstrate that auditory primary afferents can and do display rhythmic bursting patterns at stages 39-43. In our view, most of the rhythmically bursting cells are likely cochlear afferents, including unlabeled cells that did not respond to sound. This is based primarily on the fact that auditory primary afferents are by far the most numerous cells in the statoacoustic ganglion ( 8000) (Fischer et al., 1994), followed in number by lagenar cells $(\sim 2000)$ and then efferent fibers $(<400)$. Auditory cells, by virtue of their numbers, are the easiest to isolate for recording and have large cell bodies in the ganglion. Efferent fibers are not only relatively few in number but also generally very small and extremely difficult to isolate. Moreover, there are no efferent cell bodies in the periphery. Lagenar afferents, although much smaller in number, are of comparable size and have cell bodies in the peripheral ganglion that occupy somewhat different regions than those of cochlear cells. We do not have positive evidence that any of the bursting neurons were lagenar. However, we cannot rule out the possibility that some of the bursting cells were lagenar primary afferents.

The present study shows that at developmental stages 39-43 in the chicken embryo, statoacoustic ganglion cells also display slow overall spontaneous firing rates that are substantially lower than those published for stages 44-45 (Jones and Jones, 2000). Discharge rates for the youngest embryos (stages 39-41) are significantly lower than rates for older embryos (stages 42-43). Slow discharge rates are accompanied by higher CVs and longer dead times. Moreover, for stages 39 and 40, a substantial proportion of cells that we encountered were silent. These findings reveal the immature functional status of the neuroepithelium several days after the formation of synaptic contacts between hair cells and primary afferents. Indeed, our youngest embryos were recorded during the period in which compound responses to intense sound appear for the first time (above $105 \mathrm{~dB}$ SPL; E12-E13) (Saunders et al., 1973). In the youngest animals of the present study, the cells tested were unresponsive to intense tones (as high as 103-107 dB SPL) at all frequencies. It is clear that there are a limited number of neurons transmitting information about natural environmental sounds during this period. Although spontaneous synaptic activity is likely present, our findings suggest that most natural sounds are well below the threshold of afferents. From this standpoint, the period between E13 and E15 (stages 38-40) may be considered "prehearing."

As noted above, in the youngest embryos, many cells appeared to exhibit no spontaneous activity, and yet long spike trains were readily produced with nanoampere levels of current injection in each case. Romand (1984) also noted silent neurons in very young kittens. The spontaneous rate of discharge may be so low in these neurons that periods much longer than several minutes are required to resolve it. Indeed, the proportion of silent cells increased markedly in animals at stage 41 and younger; this increase was accompanied by dramatic decreases in discharge rates generally. In animals at stage 39, it was difficult to find spontaneously active cells at all. From the standpoint of developing spontaneous discharge therefore, stages 38-39 may represent periods in which activity in statoacoustic ganglion cells first emerges.

Structural immaturity may contribute to the high thresholds and immature activity patterns of stages 38-40. A considerable amount of information has been published regarding this early period of cochlear ontogeny in the chicken (Cohen and Fermin, 1978, 1985; Ard and Morest, 1984; Fermin and Cohen, 1984a,b; Whitehead and Morest, 1985a,b; Cohen and Cotanche, 1992; Cohen and Hersing, 1993; Sokolowski and Cunningham, 1999). Primary afferent synaptic contacts on hair cells are clearly present but retain immature characteristics throughout the period. Hair cells of the avian basilar papilla are organized differently than the inner and outer hair cells of mammals. However, on the basis of morphology and innervation patterns, tall and short hair cells of the avian papilla have been viewed as analogs of mammalian inner and outer hair cells, respectively. In Aves, primary afferent synapses are dominant on tall hair cells and these afferents likely provide the primary input to the brain. In contrast, efferent innervation dominates on short hair cells, and short hair cells may perform functions more related to the tuning characteristics of the basilar membrane (Manley, 1990). Although this may be true in the mature cochlea, this arrangement only gradually appears in the chicken embryo. Early differentiation of tall and short hair cells begins at stages 37-38 and continues until hair cell types and supporting cells are clearly distinguished by stage 42. Primary afferents begin to myelinate, and they manifest an immature distribution of dendrites on the papilla with nearly equal afferent innervation of tall and short hair cells during this period. The nascent tectorial membrane changes from a thin sheet of uncondensed fibrillar material at stages 37 and 38 to an emerging honeycomb appearance by stage 40 . Moreover, the middle ear is fluid-filled throughout, and ossification of the columella only begins during this period. Although efferent fibers are present, their innervation patterns and distributions are quite immature. This period also precedes the emergence in hair cells of calciumactivated $\mathrm{K}$ channels thought to be critical for sharply tuned auditory selectivity (Fuchs and Sokolowski, 1990).

The origin of the cochlear bursting pattern is unknown. The rhythmic process does not simply reflect slower discharge rates. If that were the case, then presumably $\mathrm{CVs}$ of bursting neurons would not be altered. Mature spontaneous discharge patterns exhibit a quasi-Poisson spike interval distribution reflecting a primary stochastic or random excitatory process (Kiang, 1965). As a result, spike interval CVs of mature auditory neurons are very near 1.0. This is true also in embryos at stages 44 and 45 , provided they do not exhibit bursting patterns (Jones and Jones, 
2000). Bursting represents a deviation in the stochastic pattern that manifests as a higher CV. Simply slowing the rate of discharge of an auditory neuron without altering its stochastic nature will not change the CV. Presumably therefore, bursting reflects the presence of an excitatory process fundamentally different in its temporal dynamics than the steady quasi-stochastic process found in mature animals. Hypothetically, bursting rhythms could be produced directly by the embryonic afferent neuron itself (Spitzer and Ribera, 1998), by hair cells, or both. A more indirect role for efferent neurons also cannot be ruled out. Sokolowski and Cunningham (1999) have suggested that the presence of slow, broad calcium spikes in hair cells as early as E12 (Fuchs and Evans, 1990; Fuchs and Sokolowski, 1990) could form the basis for bursting excitatory patterns. However, explicit tests of these hypotheses remain for future studies.

Primordial rhythmic activity has been shown to be of critical import to the development of normal binocular vision (Shatz, 1996). The discovery of similar discharge patterns in the periphery of the auditory system is remarkable and suggests the possibility that such rhythms could serve as a patterning signal for the auditory system during development. Recent ablation studies in the rat (Gabriele and Henkel, 2000) and altered acoustic environments in mice (Sanes and Constantine-Paton, 1985) have provided evidence for some cochlear role in central refinements. Rhythmic activity patterns in primary afferents, such as those reported here, could conceivably provide each ear with a unique activity input signature. This input signature could guide developmental refinements in central binaural processing circuits that serve the functions of sound localization and integration.

\section{REFERENCES}

Ard MD, Morest DK (1984) Cell death during development of the cochlear and vestibular ganglia of the chick. Int J Dev Neurosci 2:535-547.

Cohen GM, Cotanche DA (1992) Development of the sensory receptors and their innervation in the chick cochlea. In: Development of auditory and vestibular systems 2 (Romand R, ed), pp 101-138. Amsterdam: Elsevier.

Cohen GM, Fermin CD (1978) The development of hair cells in the embryonic chick's basilar papilla. Acta Otolaryngol 86:342-358.

Cohen GM, Fermin CD (1985) Development of the embryonic chick's tectorial membrane. Hear Res 18:29-39.

Cohen GM, Hersing W (1993) Development of the chick's auditory ossicle, the columella. Physiologist 36:S75-S76.

Constantine-Paton M, Cline HT, Debski E (1990) Patterned activity, synaptic convergence, and the NMDA receptor in developing visual pathways. Annu Rev Neurosci 13:129-154.

Fermin CD, Cohen GM (1984a) Developmental gradients in the embryonic chick's basilar papilla. Acta Otolaryngol 97:39-51.

Fermin CD, Cohen GM (1984b) Development of the embryonic chick's statoacoustic ganglion. Acta Otolaryngol 98:42-52.

Fischer FP, Eisensamer B, Manley GA (1994) Cochlear and lagenar ganglia of the chicken. J Morphol 220:71-83.

Fuchs PA, Evans MG (1990) Potassium currents in hair cells isolated from the cochlea of the chick. J Physiol (Lond) 429:529-551.
Fuchs PA, Sokolowski BHA (1990) The acquisition during development of Ca-activated potassium currents by cochlear hair cells of the chick. Proc R Soc Lond B Biol Sci 241:122-126.

Gabriele ML, Henkel CK (2000) Changes in the development of afferent patterns in the inferior colliculus of the rat following unilateral cochlear ablation. Assoc Res Otolaryngol Abs 625:180.

Galli L, Maffei L (1988) Spontaneous impulse activity of rat retinal ganglion cells in prenatal life. Science 242:90-91.

Gummer AW, Mark RF (1994) Patterned neural activity in brain stem auditory areas of a prehearing mammal, the tammar wallaby Macropus eugenii. NeuroReport 5:685-688.

Hamburger V, Hamilton HL (1951) A series of normal stages in the development of the chick embryo. J Morphol 88:49-92.

Jones SM, Jones TA (1995a) Neural tuning characteristics of auditory primary afferents in the chicken embryo. Hear Res 82:139-148.

Jones SM, Jones TA (1995b) The tonotopic map in the embryonic chicken cochlea. Hear Res 82:149-157.

Jones TA, Jones SM (2000) Spontaneous activity in the statoacoustic ganglion of the chicken embryo. J Neurophysiol 83:1452-1468.

Kiang NYS (1965) Discharge patterns of single fibers in the cat's auditory nerve. Cambridge, MA: MIT.

Lippe WR (1994) Rhythmic spontaneous activity in the developing avian auditory system. J Neurosci 14:1486-1495.

Lippe WR (1995) Relationship between frequency of spontaneous bursting and tonotopic position in the developing avian auditory system. Brain Res 703:205-213.

Manley GA (1990) Peripheral hearing mechanisms in reptiles and birds, pp 206-252. Berlin: Springer.

Masland RH (1997) Maturation of function in the developing rabbit retina. J Comp Neurol 175:275-286.

Meister M, Wong ROL, Baylor DA, Shatz CJ (1991) Synchronous bursts of action potentials in ganglion cells of the developing mammalian retina. Science 252:939-943.

Moller AR (1970) The use of correlation analysis in processing neuroelectric data. Prog Brain Res 33:87-100.

Mooney R, Penn AA, Gallego R, Shatz CJ (1996) Thalamic relay of spontaneous retinal activity prior to vision. Neuron 17:863-874.

Richter C, Sauer G, Hoidis S, Klinke R (1996) Development of activity patterns in auditory nerve fibres of pigeons. Hear Res 95:77-86.

Romand R (1984) Functional properties of auditory-nerve fibers during postnatal development in the kitten. Exp Brain Res 56:395-402.

Sanes DH, Constantine-Paton M (1985) The sharpening of frequency tuning curves requires patterned activity during development in the mouse, Mus musculus. J Neurosci 5:1152-1166.

Saunders JC, Coles RB, Gates GR (1973) The development of auditory evoked responses in the cochlea and cochlear nuclei of the chick. Brain Res 63:59-74.

Shatz CJ (1990) Impulse activity and the patterning of connections during CNS development. Neuron 5:745-756.

Shatz CJ (1996) Emergence of order in visual system development. Proc Natl Acad Sci USA 93:602-608.

Sokolowski BHA, Cunningham AM (1999) Patterns of synaptophysin expression during development of the inner ear in the chick. J Neurobiol 38:46-64.

Spitzer NC, Ribera AB (1998) Development of electrical excitability in embryonic neurons: mechanisms and roles. J Neurobiol 37:190-197.

Whitehead MC, Morest DK (1985a) The development of innervation patterns in the avian cochlea. Neuroscience 14:255-276.

Whitehead MC, Morest DK (1985b) The growth of cochlear fibers and the formation of their synaptic endings in the avian inner ear: a study with the electron microscope. Neuroscience 14:277-300.

Wong ROL, Meister M, Shatz CJ (1993) Transient period of correlated bursting activity during development of the mammalian retina. Neuron 11:923-938.

Wong ROL, Chernjavsky A, Smith SJ, Shatz CJ (1995) Early functional neural networks in the developing retina. Nature 374:716-718. 